\title{
Multi-Wavelength Regeneration of Phase Encoded Signals based on Phase Sensitive Amplifiers
}

\author{
Stylianos Sygletos, Paola Frascella ${ }^{1}$, Fatima C. Garcia Gunning, Andrew D. Ellis \\ Tyndall National Institute and Department of Physics, University College Cork, Ireland \\ Tel: +353 (21) 490 4873, Fax: +353 (21) 490 4880, e-mail: stylianos.sygletos@tyndall.ie
}

\begin{abstract}
Future high capacity optical links will have to make use of frequent signal regeneration to enable long distance transmission. In this respect, the role of all-optical signal processing becomes increasingly important because of its potential to mitigate signal impairments at low cost and power consumption. More substantial benefits are expected if regeneration is achieved simultaneously on a multiple signal band. Until recently, this had been achieved only for on-off keying modulation formats. However, as in future transmission links the information will be encoded also in the phase for enhancing the spectral efficiency, novel subsystem concepts will be needed for multichannel processing of such advanced signal formats. In this paper we show that phase sensitive amplifiers can be an ideal technology platform for developing such regenerators and we discuss our recent demonstration of the first multi-channel regenerator for phase encoded signals.
\end{abstract}

\section{INTRODUCTION}

The explosive growth of the internet traffic in the recent years, due to the proliferation of broadband services, has necessitated the development of new optical network infrastructures that can support the transmission of high capacity data volumes [1]. However, as networks continue to grow in terms of both data rates and transmission distances, the impairments from amplification, optical filtering and fibre non-linearities on the signal are becoming more critical and regeneration techniques will be required to improve the reach and the operating margins of the systems [2]. At this point a choice needs to be made between optoelectronic and all-optical regeneration technologies. Opto-electronic regenerators are highly matured, however, they cannot compete the low cost and the energy efficiency of their all-optical counterparts. On the other hand, all-optical regenerators have still a number of challenges to overcome before becoming viable and attractive solutions in next generation networks. Most important challenges are the ability to support the recently emerged phase encoded modulation formats [3] by providing efficient compensation of the accumulated phase distortions, as well as, the multiwavelength channel processing, e.g. through the use of a common nonlinear medium [4].

Until recently, all-optical regenerators were mostly designed to suppress amplitude distortion and initial schemes for phase encoded signals focused on phase preserving amplitude regeneration [5] or phase-toamplitude format conversion and subsequent amplitude regeneration [6]. Recently, direct removal of the phase distortion by exploiting the unique phase squeezing capabilities of a phase sensitive amplifier (PSA), has been demonstrated [7]. Besides, suppression of the amplitude distortion could be achieved by operating the PSA in the saturation regime [8]. Initial regenerative schemes with PSAs were based on single pump degenerate four-wave mixing (FWM) in a fiber interferometer [7]. Phase regeneration using dual pump degenerate FWM process was shown firstly in [8] and by adding the phase locking scheme proposed in [9], the first practical PSA based regenerator for differential phase shift keying signals (DPSK) has been demonstrated in [10]. On the other hand, multi-wavelength regeneration has been demonstrated to date only for on-off keying (OOK) signal formats [4]. The proposed techniques achieved amplitude noise suppression simultaneously on multiple channels either through soliton compression techniques [11],[12], or through redesign of the Mamyshev's regenerator employing carefully engineered dispersive walk-through maps to minimize the crosstalk of neighboring channels [14][15].

In this paper we discuss the results of our recent demonstration of the first multi-channel regenerator of phase encoded signals [16]. Specifically, using a novel PSA based scheme we managed to achieve reduction of phase distortion simultaneously on two independent $42.66 \mathrm{Gbit} / \mathrm{s}$ DPSK input signals enabling a regeneration in terms of receiver sensitivity of more than $10 \mathrm{~dB}$ on both channels.

\section{EXPERIMENTAL SETUP}

The experimental setup of the proposed regeneration scheme is illustrated in Error! Reference source not found.. Two independently modulated DPSK signals at 42.66Gbit/s have been considered at the input, produced by modulating separate lasers sources, at $1549.91 \mathrm{~nm}$ and $1550.71 \mathrm{~nm}$, with a pseudorandom binary sequence (PRBS) of $2^{31}-1$ pattern. Phase distortion was added to both channels using a phase modulator driven by a 14 $\mathrm{GHz}$ square wave from an independent device. The two channels were subsequently amplified to $14 \mathrm{dBm}$ by an Erbium doped fibre amplifier (EDFA) and launched into the phase regenerator.

\footnotetext{
${ }^{1}$ now with Nokia Siemens Networks Portugal, S.A., Lisbon, Portugal
} 

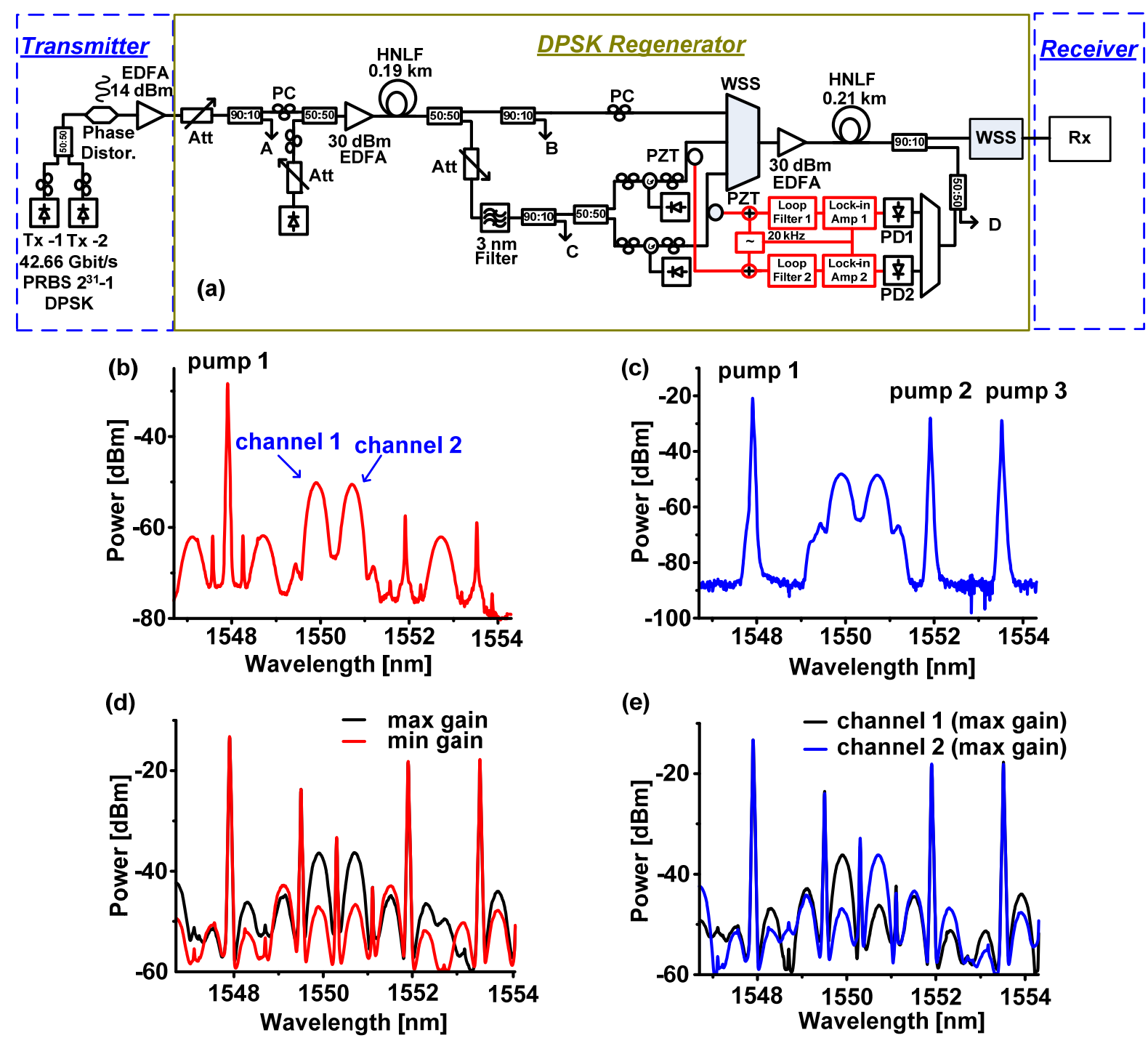

Fig. 1 (a) Experimental setup of the two channels PSA based phase regenerator for BPSK signals. (b) Optical spectrum after the carrier extraction stage; (Tx: transmitter, PC: polarization controller, Att: attenuator, PD: photodiode, Rx: receiver). (c) Optical spectrum at the input of the $2^{\text {nd }}$ HNLF. (d) Optical spectrum at the output of the $2^{\text {nd }}$ HNLF (point D) when both channels are locked either at the maximum or minimum phase sensitive gain state of the PSA. (e) Optical spectrum at the output of the $2^{\text {nd }}$ HNLF (point D) when the channels are locked at different phase sensitive gain states of the PSA.

The subsystem was an implementation of an inline PSA consisting of a phase synchronization and a phase regeneration stage. At the phase synchronization stage the two DPSK channels were mixed with a strong CW pump signal at $1547.91 \mathrm{~nm}$ in a highly nonlinear fiber (HNLF) generating optical carriers at symmetric wavelength positions see Fig. 1(b). The extracted carriers were also self-locked to the corresponding data signal and the common local pump so that the necessary conditions for PSA performance were maintained among the interacting waves. Subsequently, the carriers were used to injection lock two local lasers providing clean and high power pumps for the regeneration stage of the PSA. The two injection locked pump lasers from one path and the signal and common pump from the other were directed to a wavelength selective switch (WSS). The WSS suppressed the unwanted spectral components that had been generated from the FWM process in the carrier extraction stage, see Fig. 1(c). After the WSS, the combined signals were amplified and launched into a second HNLF for the phase regeneration. Since the experimental setup was vulnerable to slow temperature induced path length variations, which affected the phase sensitive gain of the PSA separately on each DPSK channel, compensation against the corresponding phase drift was required. This was achieved with a feedback circuit setup that used both channels at the output of the PSA as error signals to control two different piezoelectric fibre stretchers (PZTs), one for each pump path. Each channel could be independently locked at the maximum or minimum phase sensitive gain states of the PSA. Error! Reference source not found.(d) depicts the resulting spectra at the output of the regeneration stage of the PSA, taken at point D, when the channels were simultaneously locked at the maximum or minimum phase sensitive gain, respectively. A gain contrast ratio of 
$11 \mathrm{~dB}$ was achieved for both channels. Error! Reference source not found.(e) depicts the output spectra when the two channels were locked at two different phase sensitive gain states.

The interaction of the three pumps in the HNLF created strong FWM products that could potentially overlap with the incoming signals. A careful selection of the frequency distance of the common pump with each signal to be a non-integer multiple of their channel spacing, allowed us to avoid such scenario, see Fig. 1(d), (e). A second WSS at the output of the PSA was also required to filter the two signals and remove the unwanted wavelengths. Finally, at the receiver section an 1-bit delay interferometer was used for demodulating the channels, and a single-ended photodiode and phase locked loop clock recovery circuit was used to detect the signal allowing for BER analysis.

\section{RESULTS}

A rigorous optimization of each PSA stage has been carried out to maximize the overall performance. For the carrier extraction, the optimum power level of the local pump with respect to the power of the input signal had to be optimized so that the carriers could be extracted without degrading the signal quality. Bit error rate measurements at the output of the $1^{\text {st }}$ HNLF revealed that the pump-to-input signal power ratio should be larger than $3 \mathrm{~dB}$ to maintain a sensitivity penalty of less than $0.5 \mathrm{~dB}$ for both channels.

Similarly at the phase regeneration stage, the optimum power level of the local pumps and signal powers has been optimized. In Fig. 2(a-b) the measured penalty in terms of receiver sensitivity for the two channels at the output of the PSA is depicted, as well as, the corresponding improvement when the channels were phase distorted by $3 \mathrm{~dB}$ at the PSA input. In Fig 2(a) the measurements have been taken as a function of the ratio of their common pump (pump1) power to the mutual power level of the other pump waves (pump 2, pump 3) measured at the output of the $2^{\text {nd }}$ HNLF fibre. The common pump interacted with both pump 2 and pump 3, and at low power ratios it was strongly depleted. As a result the effective noise figure of the PSA was increased and an additional sensitivity penalty was introduced. The optimum operating region was achieved for ratios of around $5 \mathrm{~dB}$, where the sensitivity penalty was less than $0.5 \mathrm{~dB}$ for both channels. At the same point best suppression of the introduced phase distortion was achieved enabling a $2 \mathrm{~dB}$ improvement in the received sensitivity of the two channels.

Error! Reference source not found.2(b) shows the sensitivity penalty for both channels as a function of the ratio of pump-1 power to the common power level of the two DPSK signals. At low ratio values the regenerator operated in strong saturation and due to the enhanced nonlinear interaction of the data signals with the common pump the PSA performance was slightly degraded. The degradation was significantly stronger when the pumpto-signal ratio exceeded $12 \mathrm{~dB}$, by attenuating the two signals, due to the corresponding increase in the effective noise figure of the PSA. The optimum point appeared at the value of $8 \mathrm{~dB}$, and resulted in a penalty of $0.5 \mathrm{~dB}$. At the same point the PSA presented maximum regenerative capability by improving by $2 \mathrm{~dB}$ the sensitivity of phase distorted input channels.
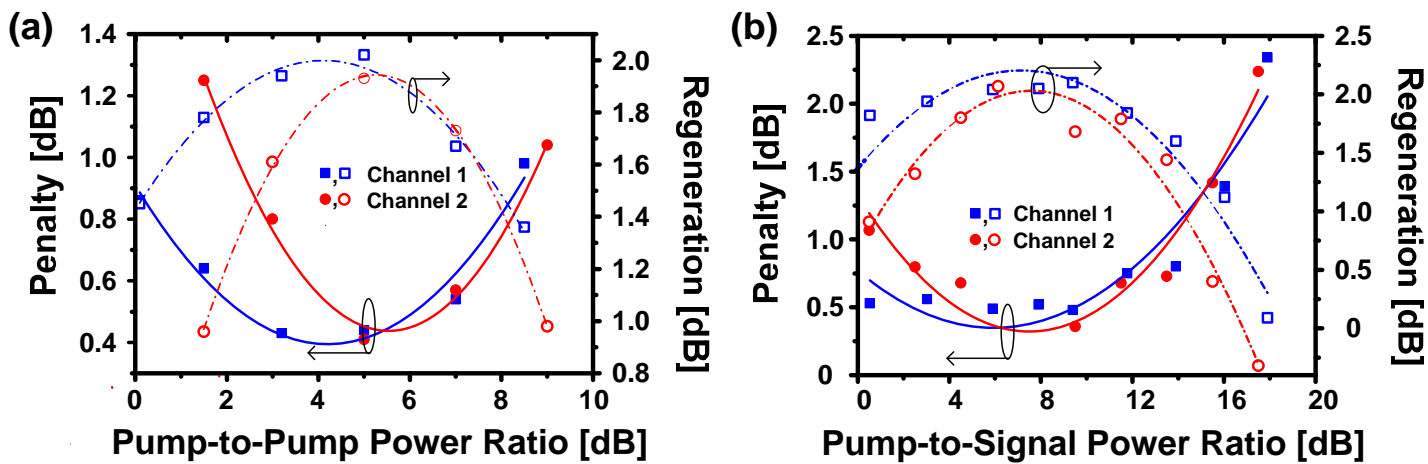

Fig. 2 Receiver sensitivity penalty or improvement with respect to back-to-back performance measured for both channels at the output of the PSA, (a) as a function of the pump-to-pump power ratio for undistorted channels at the PSA input as well as when they have experienced $3 \mathrm{~dB}$ penalty due to phase distortion, (b) as a function of the pump-to-signal power ratio for undistorted channels at the PSA inputs well as when they have experienced $3 \mathrm{~dB}$ penalty due to phase distortion.

Finally, Fig. 1(a)-(b) depict measured BER curves for both channels taken against total received power, for input degradations of about $\sim 10 \mathrm{~dB}$ at $10^{-9}$. It is also shown that in the absence of any input degradation the receiver sensitivity penalty of each channel at $10^{-9}$ was less than $1 \mathrm{~dB}$, and error free operation was achieved. Furthermore, when introducing significant phase distortion on the input channels, the PSA demonstrated the anticipated phase squeezing capabilities. The restored eye diagrams, illustrated in Fig. 13(c) and Fig. 13(d), prove the high regenerative efficiency of our developed scheme. 

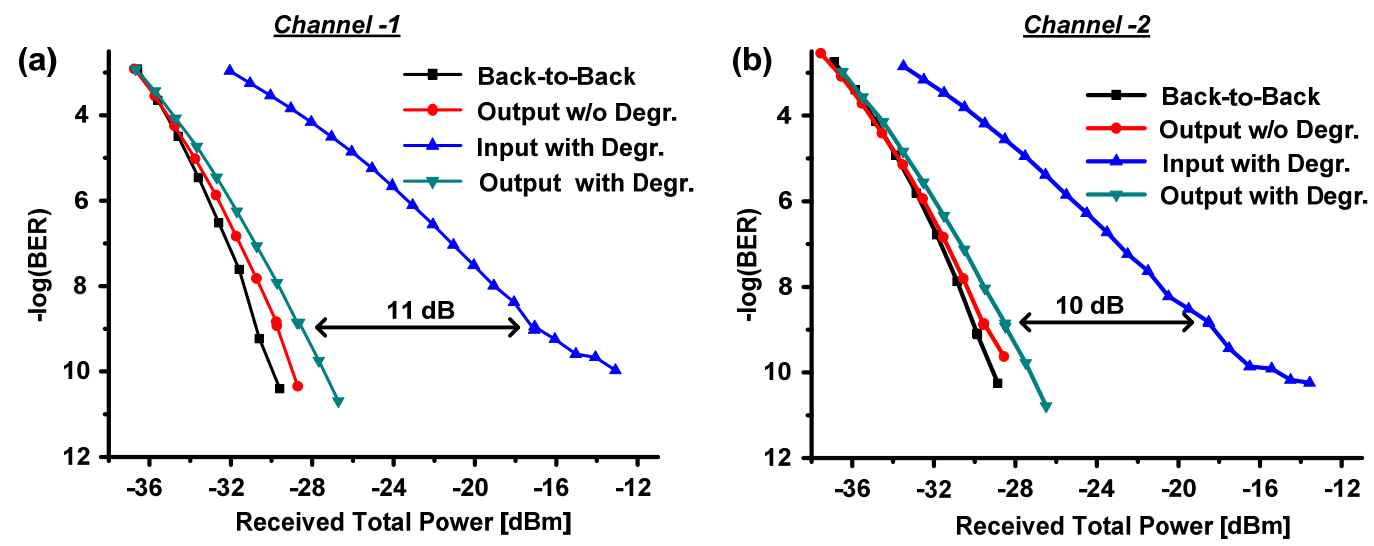

(c)
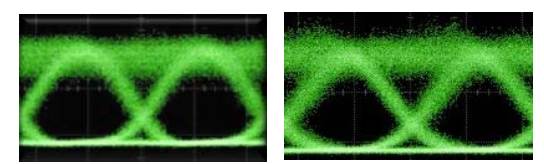

(d)

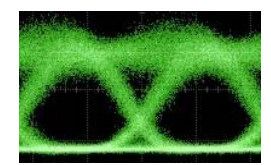

Back-to-Back
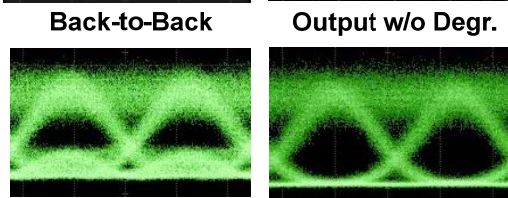

Input with Degr.

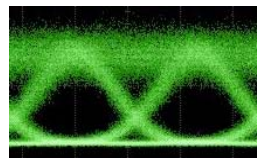

Output with Degr.

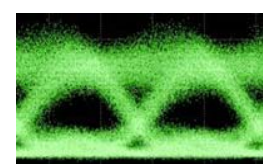

Input with Degr.

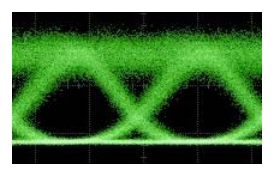

Output w/o Degr.

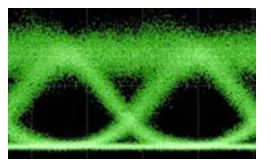

Output with Degr.

Fig. 1: BER measurements versus total received power (a) for channel 1 and (b) for channel 2 at input/output of PSA with/without the presence of input periodic degradation. Corresponding eye diagrams (c) for channel 1 and (d) for channel 2

\section{CONCLUSIONS}

We have discussed our recent experimental implementation of an in-line PSA with multichannel regenerative capabilities. The regenerative performance of our scheme has been evaluated against periodic phase distortion on two $42.66 \mathrm{Gbit} / \mathrm{s}$ DPSK signals. Significant recovery of the undistorted signal quality has been achieved for both channels, quantified by more than $10 \mathrm{~dB}$ improvement in terms of receiver sensitivity.

\section{ACKNOWLEDGEMENTS}

This work has been supported and by European Communities Seventh Framework Programme FP/2007-2013 under grant agreement 224547 (PHASORS) and by Science Foundation Ireland under Grant 06/IN/I969

\section{REFERENCES}

[1] A. D. Ellis, J. Zhao, D. Cotter, “Approaching the non-linear Shannon limit,” IEEE J. Lightwave Technol. 28, 423-433, (2010).

[2] S. J. Ben Yoo, “All-optical regeneration for ultra-long fiber links and its prospects for future applications in new modulation formats," in Proc OFC 2009, paper OThS4.

[3] P. J. Winzer, R.-J. Essiambre, “Advanced optical modulation formats,” Proceedings of IEEE, 94, 952-984, (2006)

[4] F. Parmigiani, L. Provost, P. Petropoulos, D. J. Richardson, W. Freude, J. Leuthold, A. D. Ellis, I. Tomkos, "Progress in multichannel all-optical regeneration based on fiber technology,” IEEE J. Select. Topics in Quantum Electronics vol. 18, no. 2, March 2012

[5] M. Matsumoto and K. Sanuki, "Performance improvement of DPSK signal transmission by a phase preserving amplitude limiter," Optics Express 15, 8094-8103, (2007).

[6] P. Vorreau, et al, “Cascadability and regenerative properties of SOA all-optical DPSK wavelength conversion,” IEEE Photon. Technol. Lett. 18, 1970-1973 (2006).

[7] K. Croussore, C. Kim, and Guifang. Li, “All-optical regeneration of differential phase-shift keying signals based on phase-sensitive amplification,” Opt. Lett. 29, 2357-2359 (2004).

[8] K. Croussore and Guifang Li, "Phase regeneration of NRZ-DPSK singals based on symmetric-pump phase sensitive amplification," IEEE Photon. Technol. Lett.19, 864-864 (2007).

[9] R. Weerasuriya, et al, “Generation of frequency symmetric signals from a BPSK input for phase sensitive amplification,” in Proc. OFC 2010, OWT6,San Diego CA, USA, 21-25 March 2010

[10] R. Slavík,et al., “All-optical phase and amplitude regenerator for next generation telecommunications systems,” Nature Photonics 4, 690-695 (2010)

[11] T. Ohara, H. Takara, A. Hirano, K. Mori, S. Kawanishi, “40-Gb/s × 4-channel all-optical multichannel limiter utilizing spectrally filtered optical solitons,” IEEE Photon. Technol. Lett. 15, 763-765, (2003)

[12] B. Cuenot, A. D. Ellis, "WDM signal regeneration using a single all-optical device,” Optics Express 15, 11492-11499, (2007)

[13] P. V. Mamyshev, "All-optical data regeneration based on self-phase modulation effect," in Proc ECOC 1998, $475-476$

[14] M. Vasilyev, T. I. Lakoba, “All-optical multichannel 2R regeneration in a fibre-based device,” Opt. Lett. 30, 1458-1460, 2005

[15] L. Provost, F. Parmigiani, K. Mukasa, M. Takahashi, J. Hiroishi, M. Tadakuma, P. Petropoulos, D. J. Richardson, "Simultaneous alloptical 2R regeneration of $4 \times 10 \mathrm{Gbit} / \mathrm{s}$ wavelength division multiplexed channels,” in Proc. ECOC 2007, Di 4.5.1.

[16] P. Frascella, S. Sygletos, S. K. Ibrahim, L. Grüner-Nielsen, R. Phelan, J. O’Gorman, A. D. Ellis, “Two channel phase regeneration based on a "black-box" Phase Sensitive Amplifier," in Proc. ECOC 2011, Th. 11. LeCervin.2 